\title{
Harmonic Contribution Determination of Multiple Harmonic Sources Based on FastICA in Distribution Network
}

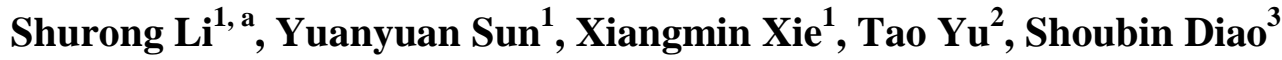 \\ ${ }^{1}$ School of Electrical Engineering, Shandong University, Jingshi road, Jinan 250061, China \\ ${ }^{2}$ Jinan Urban Planning and Design Institute, Shunhua road, Jinan 250101, China \\ ${ }^{3}$ State Grid Licheng Power Supply Company, Hualong road, Jinan 250001, China \\ a18663794279@163.com
}

Keywords: harmonic contribution determination; multiple harmonic sources; power quality

Abstract: The harmonic pollution at the point of common coupling (PCC) in the public grid is the outcome of combined contribution of multiple harmonic sources. For a better determination of harmonic contribution, this paper proposes a method to quantify harmonic contributions of multiple harmonic sources based on FastICA. The background harmonics and the harmonic transfer impedance can be estimated accurately by the proposed method based on FastICA. Harmonic contribution of multiple harmonic source is calculated after the estimation of the background harmonics and the harmonic transfer impedance. A 18 nodes distribution network model is established in MATLAB/simulink to test this method. The results show that this method can quantify harmonic contribution of each harmonic source accurately.

\section{Introduction}

More and more non-linear loads are introduced into public grid with the high speed development of industrial technology and modern science. A large number of distributed generation (DG) connected to the public grid through inverter, which caused serious harmonic pollution [1,2] . Harmonic current injected to public grid caused by non-linear loads and power electronic switches may cause many passive impacts, such as high power lose and shorten service life [3]. Therefore, the harmonic contribution determination is significant. "non intrusive" method are the common used harmonic contribution determination method[4-7].These researches make good contribution to harmonic contribution determination(HCD), but there are still some problem need to be solved.

Fast independent component analysis (FastICA) need not to select step size and has fast convergence speed. Therefore, the FastICA has some advantages to do HCD. Some researchers have applied this technology to do HCD[8], which were proven to be effective. But the researches are only for single harmonic source issue.

A method for quantifying harmonic contributions of multiple harmonic sources based on FastICA is proposed in this paper. The proposed method obtains $h$-th harmonic phasor data by using 
FFT results of the voltage and current data measured by PMU. Then, this method separates the data into real component and imaginary component for which can satisfy the assumption of FastICA. Mixed matrix and each source signals can be obtained by FastICA. Harmonic contribution of each harmonic source is calculated by projecting harmonic voltage of PCC caused by each harmonic source on total harmonic voltage of PCC.

\section{Basic theory}

\subsection{Fast independent component analysis}

At present, ICA is the most common used method to solve BSS issue. ICA can separate mixed signals without any priori knowledge. Besides, ICA don`t destroy the details of source signals. Mathematic model of ICA is shown as equation (1):

$$
X(t)=A S(t)
$$

Where $t$ is discrete sample time, $t=1,2, \ldots, t_{\mathrm{n}} . S(t)$ is the matrix composed of $N$ unknown source signals, $S(t)=\left[S_{1}(t), S_{2}(t), \ldots, S_{N}(t)\right]^{T} . X(t)$ is the matrix composed of $M$ observed signals, $X(t)=\left[X_{1}(t), X_{2}(t), \ldots, X_{M}(t)\right]^{T}$.

FastICA algorithm makes negative entropy as the objective function evaluating the independency of the signal and uses approximate negative entropy as the index of evaluating nonGaussianity of random variable. The bigger the negative entropy is the greater the non-Gaussianity is. The objective function of FastICA algorithm adopted by this paper is a kind of negative entropy approximate calculation method based on maximum entropy principle. The mathematical expression is shown as follows:

$$
J(y) \approx K[E\{G(y)-E(G(v))\}]^{2}
$$

Where $K$ is a positive constant, $y$ and $v$ are the random variable whose mean is 0 and variance is 1. $G$ is a non-quadratic function. There we set $G$ as follow:

$$
G(y)=y^{3}
$$

The essence of FastICA algorithm is seeking the separation matrix W which makes the equation (2) largest and then calculating corresponding evaluating results Y. The source signal can't always be completely independent in reality. Therefore the data need pretreatment before blind signal separation. First step is removing mean value, which is for avoiding the errors caused by the big gap between data dimension. Second step is whitening, which is for removing the correlation between mixed signals. The equation of removing mean value is:

$$
\hat{X}\left(t_{i}\right)=X\left(t_{i}\right)-\frac{1}{n} \sum_{i=1}^{n} X\left(t_{i}\right)
$$

Where $n$ is the length of data, which should be proper. The length of data is too long or short both will cause error.

Whitening is linear conversion, which is shown as follows:

$$
Z(t)=W_{0} \hat{X}(t)
$$

Where $W_{0}$ is whitening matrix. Whitening matrix can be calculated by $W_{0}=\Lambda^{-0.5} U^{T}$, where $\Lambda$ is the eigenvalue matrix of covariance matrix $C_{X}\left(C_{X}=\hat{X} \hat{X}^{T}\right)$ and $U$ is the eigenvector matrix of $C_{X}$. The 
equation of iteration is:

$$
\left\{\begin{array}{l}
W_{i+1}=E\left\{X G\left(W_{i}^{T} Z\right)\right\}-E\left\{G^{\prime}\left(W_{i}^{T} Z\right)\right\} W_{i} \\
W_{i+2}=\frac{W_{i+1}}{\left\|W_{i+1}\right\|}
\end{array}\right.
$$

Where $i$ is number of iterations, $i=1,2, \ldots, m$. When the change of $\left\|W_{i}\right\|$ is smaller than a given value, iteration will be done.

In conclusion, the steps are shown in Figure 1:

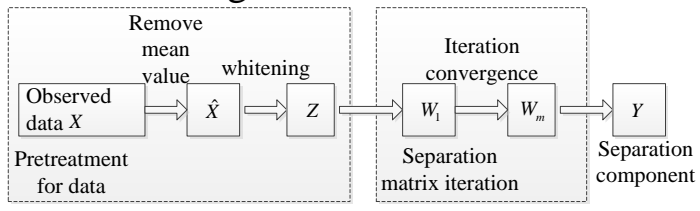

Figure 1. Steps of FastICA

\subsection{Quantifying harmonic contributions of multiple harmonic sources based on FastICA}

Harmonic pollution of multiple harmonic sources issue can be expressed as Figure 2:

As shown in Figure 2, the harmonic voltage of PCC is the outcome of combined contribution of harmonic source load and background harmonic. In distribution network, harmonic current injected by harmonic source load flow through harmonic impedance and magnify the harmonic voltage of PCC. Harmonic voltage of PCC can be calculated by equation (7) and (8):

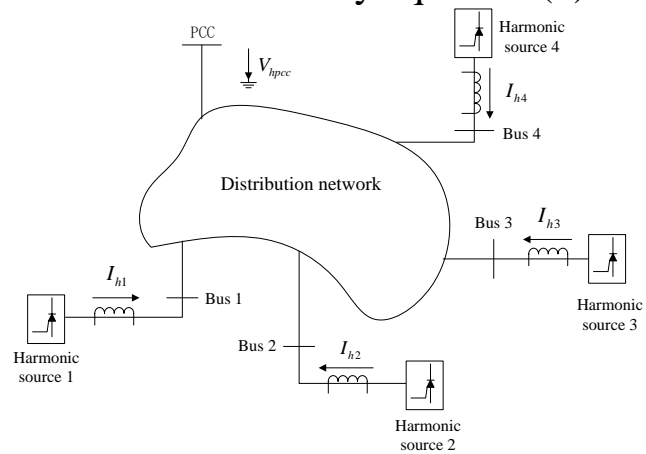

Figure 2. Harmonic pollution in distribution network

$$
\left[\begin{array}{l}
\dot{V}_{h p c c} \\
\dot{I}_{h 1} \\
\dot{I}_{h 2} \\
\dot{I}_{h 3} \\
\dot{I}_{h 4}
\end{array}\right]=\left[\begin{array}{ccccc}
\dot{Z}_{h 1 p c c} & \dot{Z}_{h 2 p c c} & \dot{Z}_{h 3 p c c} & \dot{Z}_{h 4 p c c} & 1 \\
1 & 0 & 0 & 0 & 0 \\
0 & 1 & 0 & 0 & 0 \\
0 & 0 & 1 & 0 & 0 \\
0 & 0 & 0 & 1 & 0
\end{array}\right]\left[\begin{array}{l}
\dot{I}_{h 1} \\
\dot{I}_{h 2} \\
\dot{I}_{h 3} \\
\dot{I}_{h 4} \\
\dot{V}_{h 0}
\end{array}\right] \quad \text { (7) } \underbrace{\left[\begin{array}{l}
\dot{V}_{h p c c} \\
\dot{I}_{h 1} \\
\dot{I}_{h 2} \\
\dot{I}_{h 3} \\
\dot{I}_{h 4}
\end{array}\right]}_{X}=\underbrace{\left[\begin{array}{ccccc}
k_{1} \dot{Z}_{h 1 p c c} & k_{2} \dot{Z}_{h 2 p c c} & k_{3} \dot{Z}_{h 3 p c c} & k_{4} \dot{Z}_{h 4 p c c} & k_{5} \\
k_{1} & 0 & 0 & 0 & 0 \\
0 & k_{2} & 0 & 0 & 0 \\
0 & 0 & k_{3} & 0 & 0 \\
0 & 0 & 0 & k_{4} & 0 \\
0 & \dot{I}_{h 1-Y} \\
\dot{I}_{h 2-Y} \\
\dot{I}_{h 3-Y} \\
\dot{I}_{h 4-Y} \\
\dot{V}_{h 0-Y}
\end{array}\right]}_{A}
$$

Where $\dot{I}_{h 1-Y}, \dot{I}_{h 2-Y}, \dot{I}_{h 3-Y}, \dot{I}_{h 4-Y}$ and $\dot{V}_{h 0-Y}$ are FastICA separation results. Indeterminacy of magnitude can be eliminated by giving different values to $k_{1}, k_{2}, k_{3}, k_{4}$. Indeterminacy of order can be solved by correlating the FastICA signals and mixed signals. The correlation can be estimated by equation (9).

$$
\rho_{i j}=\frac{\sum\left(\mathrm{s}_{i}-\bar{s}_{i}\right)\left(\mathrm{y}_{j}-\bar{y}_{j}\right)}{\sqrt{\sum\left(\mathrm{s}_{i}-\bar{s}_{i}\right)^{2}} \sqrt{\sum\left(\mathrm{y}_{j}-\bar{y}_{j}\right)^{2}}}
$$


Where $\rho_{i j}$ is the correlation coefficient between source signal si and separated signal yi. si is correspond to yi when $\left|\rho_{i j}\right|$ is close to 1 . The harmonic transfer impedance can be calculated after solving the indeterminacy of magnitude and order. The harmonic transfer impedance can be calculated by equation (10).

$$
\dot{Z}_{h 1 p c c}=\frac{A(1,1)}{A(2,1)}
$$

The same to $\dot{Z}_{h 2 p c c}, \dot{Z}_{h 3 p c c}, \dot{Z}_{h 4 p c c}$. Division of harmonic contributions of multiple harmonic sources is shown as Figure 3.

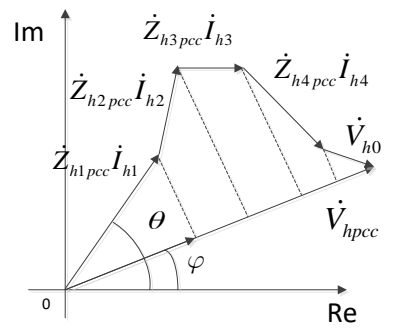

Figure 3. Calculation of harmonic contribution of multiple harmonic sources

$H$-th harmonic contribution of each harmonic source can be calculated by the projection of $h$-th harmonic voltage at the PCC caused by each harmonic source on $\dot{V}_{\text {hpcc }}$. As equation (11) shows:

$$
H I_{h 1}=\frac{\left|\dot{Z}_{h 1 p c c} \dot{I}_{h 1} \cos (\theta-\varphi)\right|}{\left|\dot{V}_{h p c c}\right|} \times 100 \%
$$

Where $H I_{h 1}$ is h-th harmonic contribution of harmonic source load 1 at the PCC. $\theta$ is the phase angle of $\dot{Z}_{h 1 p c c} \dot{I}_{h 1}$. $\varphi$ is the phase angle of $\dot{V}_{h p c c}$.

In conclusion, steps of the quantifying harmonic contributions of multiple harmonic sources based on FastICA are shown in Figure 4.

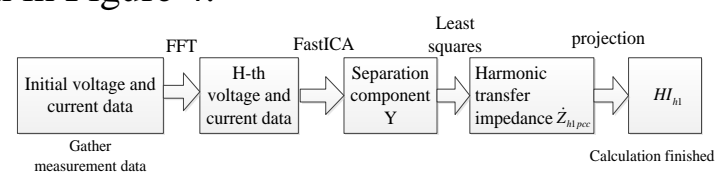

Figure 4. Steps of the quantifying harmonic contributions of multiple harmonic sources based on FastICA paper

\section{Simulation analysis}

This paper set up 18 nodes model of $10 \mathrm{kV}$ distribution network in Simulink to verify the correctness of the proposed method. Topology of distribution network is shown in Figure 5.

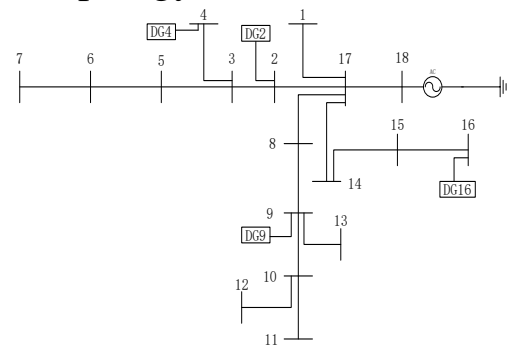

Figure 5. Topology of distribution network 
The harmonic voltage and harmonic current data are obtained by simulation. Sample frequency of simulation data is $12800 \mathrm{~Hz}$. Length of data is 256 points.

FastICA results shown as follow(5-th harmonic):

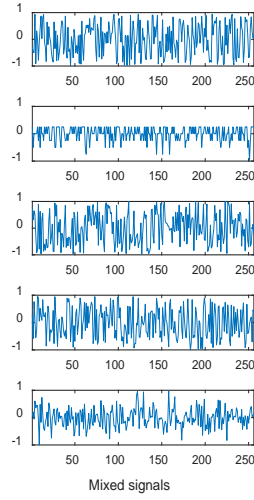

(a) real component

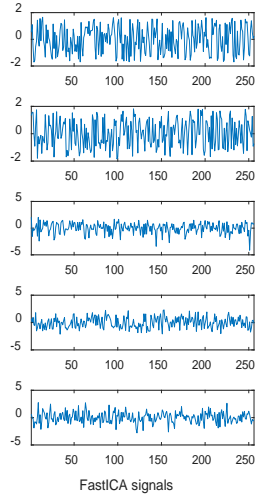

Figure 6. FastICA separation effect

It is not difficult to identify the corresponding relationship between mixed signals and FastICA signals by calculating correlation coefficients. Correlation coefficients are calculated as follow ( $\rho_{-x}$ is real component, $\rho_{-y}$ is imaginary component):

The harmonic contribution of each harmonic source at the PCC is calculated in Table 1.

$$
\rho_{-x}=\left[\begin{array}{ccccc}
0.923 & 0.015 & 0.023 & -0.011 & 0.005 \\
0.010 & 0.034 & 0.941 & 0.001 & 0.256 \\
0.132 & 0.067 & -0.031 & 0.776 & 0.163 \\
0.008 & -0.961 & 0.010 & 0.006 & 0.019 \\
0.007 & 0.016 & 0.021 & -0.003 & -0.973
\end{array}\right] \rho_{-y}=\left[\begin{array}{ccccc}
0.008 & 0.014 & 0.951 & -0.013 & 0.021 \\
0.015 & 0.007 & -0.017 & 0.946 & -0.009 \\
0.003 & -0.942 & 0.022 & 0.013 & 0.010 \\
0.981 & 0.012 & -0.008 & -0.009 & 0.013 \\
0.021 & 0.018 & -0.013 & 0.011 & 0.927
\end{array}\right]
$$

Table 1. Results of quantifying harmonic contribution (5-th harmonic)

\begin{tabular}{ccc}
\hline Harmonic source & $\begin{array}{c}\text { Accurate } \\
\text { value/\% }\end{array}$ & $\begin{array}{c}\text { Estimated } \\
\text { value/\% }\end{array}$ \\
\hline DG 2 & 13.35 & 12.86 \\
DG 4 & 27.14 & 26.31 \\
DG9 & 30.53 & 29.16 \\
DG16 & 21.94 & 19.64 \\
Background & 7.04 & 12.03 \\
harmonic & 100 & 100 \\
Total &
\end{tabular}

It can be seen from Table 1 that the largest error is $4.99 \%$ and the error of the main harmonic sources like DG 4, DG 9 and DG 16 is around just 1\%. Therefore, the proposed method is quite accurate. The results in Table 1 indicate that FastICA will play a huge role in the field of HCD for multi-harmonic sources.

\section{Conclusion}

This paper proposed a HCD method for multiple harmonic sources based on FastICA. Firstly, separate the phasor data into real component and imaginary component. Secondly, apply the real component and imaginary component to equivalent mixture model of multiple harmonic sources 
respectively. Thirdly, separate all source signals by FastICA and calculate harmonic impedance and background harmonic voltage. Lastly, project the $h$-th harmonic voltage components at PCC caused by each harmonic source to total $h$-th harmonic voltage of PCC to calculate $h$-th harmonic contribution.

The simulation study show that the proposed method is of high accuracy. Besides, the method don`t need any prior knowledge, which is easy to use. All above say that the proposed method is with high practical value.

\section{Acknowledgments}

This work is supported by the National Natural Science Foundation of China (51577108) and Young Scholars Program Shandong University (2016WLJH07).

\section{References}

[1] H. E. Mazin, W. Xu, B. Huang. “Determining the harmonic impacts of multiple harmonic-producing loads,” IEEE Transaction on Power Delivery, New York, 2011, pp. 1187-1195.

[2] L Pang. "Research for identification of harmonic source and quantification of harmonic responsibility," M.S. thesis, Hunan University, 2012.

[3] L. L. Chang, Y. Xie, P. Zeng, et al. "New integrated quality devices for wind farm," Proceedings of the CSU-EPSA, Tianjin, 2016, pp. 82-86.

[4] W. Xu, Y. Liu. "A method for determining customer and utility harmonic contribution at the point of common coupling," IEEE Transaction on Power Delivery, New York, 2000, pp. 804-811.

[5] Y. Y. Sun, Z. M. Yin. "Quantifying harmonic responsibilities of multiple harmonic sources based on M-estimation robust regression," Proceedings of the CSEE, Beijing, 2012, pp. 166-173.

[6] J. Hui, H. G. Yang, S. F. Lin, et al. "Assessing utility harmonic impedance based on the covariance characteristic of random vectors," IEEE Transaction on Power Delivery, New York, 2010, pp.1778-1786.

[7] H. C. Hua, X. F. Jia, D. S. Cao, et al. "A maximum likelihood method for harmonic impedance estimation," Proceedings of the CSU-EPSA, Tianjin, 2014, pp. 1692-1699.

[8] X. Zhao, H. G. Yang. "A new method to calculate the utility harmonic impedance based on FastICA," IEEE Transaction on Power Delivery, New York, 2016, pp. 381-388. 